\title{
A Flight Dynamics Perspective of the Orion Pad Abort One Flight Test
}

\author{
Jinu Idicula, Peggy S. Williams-Hayes*, and Ryan Stillwater*, \\ NASA Dryden Flight Research Center, Edwards, CA, 93523 \\ Lt. Max Yates* \\ Unites States Air Force, Edwards, CA, 93523
}

\begin{abstract}
The Orion Crew Exploration Vehicle is America's next generation of human rated spacecraft. The Orion Launch Abort System will take the astronauts away from the exploration vehicle in the event of an aborted launch. The pad abort mode of the Launch Abort System will be flight-tested in 2009 from the White Sands Missile Range in New Mexico. This paper examines some of the efforts currently underway at the NASA Dryden Flight Research Center by the Controls \& Dynamics group in preparation for the flight test. The concept of operation for the pad abort flight is presented along with an overview of the guidance, control and navigation systems. Preparations for the flight test, such as hardware testing and development of the real-time displays, are examined. The results from the validation and verification efforts for the aerodynamic and atmospheric models are shown along with Monte Carlo analysis results.
\end{abstract}

\section{Nomenclature}

$\alpha \quad$ Angle of Attack (deg)

$\beta \quad$ angle of sideslip, deg)

AA-1 Ascent Abort 1

AA-2 Ascent Abort 2

AA-3 Ascent Abort 3

ACM Attitude Control Motor

AGL above ground level

AIL article-in-the-loop

AM Abort Motor

ANTARES Advanced NASA Technology Architecture for Exploration Studies

API Application Programming Interface

c.g. center of gravity

CAP CEV Aerosciences Project

CEV Crew Exploration Vehicle

CM Crew Module

DFI Developmental Flight Instrumentation

DOF degrees of freedom

FADS Flush Air Data System

FTA Flight Test Article

GN\&C guidance, navigation, and control

GPS Global Positioning System

GRAM Global Reference Atmospheric Model

IADS Interactive Analysis and Display Software

INS Inertial Navigation System

*Aerospace Engineer, Controls \& Dynamics Branch, M/S 4840D, AIAA Member. 


$\begin{array}{ll}\text { JM } & \text { Jettison Motor } \\ \text { LAS } & \text { Launch Abort System } \\ \text { LAV } & \text { Launch Abort Vehicle } \\ \text { MC } & \text { Monte Carlo } \\ \text { MOF } & \text { Mobile Operations Facility } \\ \text { OFI } & \text { Operational Flight Instrumentation } \\ \text { p } & \text { roll rate, deg/s } \\ \text { PA-1 } & \text { Pad Abort 1 } \\ \text { PA-2 } & \text { Pad Abort 2 } \\ \text { PID } & \text { Proportional Integral Derivative } \\ \text { q } & \text { pitch rate, deg/s } \\ \text { r } & \text { yaw rate, deg/s } \\ \text { RRA } & \text { Range Reference Atmosphere } \\ \text { SIGI } & \text { Space Integrated GPS/INS } \\ \text { SM } & \text { Service Module } \\ \text { TAR3 } & \text { Treatment Learner } \\ \text { UHF } & \text { Ultra High frequency } \\ \text { V\&V } & \text { validation and verification } \\ \text { VMC } & \text { Vehicle Management Computer } \\ \text { WSMR } & \text { White Sands Missile Range }\end{array}$

\section{Introduction}

7 HE Orion Crew Exploration Vehicle (CEV) is America's next generation of human rated spacecraft for returning astronauts to the moon and beyond. Orion is heavily influenced by the Apollo spacecraft albeit is much larger in size and incorporates the latest advances in aerospace technology. Orion is comprised of three modules: the Crew Module (CM), the Service Module (SM) and the Launch Abort System (LAS). The CM houses the astronauts. The SM, which is not a part of this flight test, will provide power and propulsion to the spacecraft while in orbit. The LAS is the Orion safety system, that, in the event of an aborted launch, will take the astronauts to safety. The LAS has the capability to abort from the launch pad as well as during ascent at medium and high altitudes. To ensure that this system can be used safely, these three abort modes will be demonstrated through various flight tests over the next several years. Currently there are two pad (Pad Abort 1, PA-1, and Pad Abort 2, PA-2) and three medium altitude (Ascent Abort 1, AA-1, Ascent Abort 2, AA-2, and Ascent Abort 3, AA-3) abort tests planned.

The scope of this paper is to provide an overview of the efforts leading up to the flight test of PA-1 by the Controls \& Dynamics group at the NASA Dryden Flight Research Center (Edwards, California). Some of these tasks include: assessing the sensitivities of the vehicle using Monte Carlo analysis, running off-nominal trajectories, and supporting the verification and validation (V\&V) efforts. The PA-1 flight test is tentatively scheduled for a late 2009 launch from Launch Complex 32 East at the White Sands Missile Range (WSMR) in New Mexico. It is important to note that the vehicle to be flight-tested, known as the Flight Test Article (FTA), will be different from the Orion CEV in a number of ways. For example, the FTA is an unmanned vehicle and the avionics architecture will be a prototype of the production vehicle. The primary focus of the PA-1 flight test is not to test a one-to-one vehicle but is rather to advance flight systems maturity, test data acquisition systems, and validate simulations and models. The LAS concept of operations will be presented along with an examination of the LAS hardware. Article-in-the loop (AIL) testing will be described and an overview of the flight-test operations will be given. Finally, simulations, tools, and analysis techniques used to study the PA-1 trajectory will be described in detail.

\section{Pad Abort 1 Vehicle Overview}

\section{A. Pad Abort 1 Concept of Operations}

The Launch Abort System along with Orion as an integrated stack are shown in Fig. 1. The coordinate system used in this paper is as follows; the roll axis points positive out of the nose on the centerline of the vehicle, the pitch axis is positive going to the right from the centerline and the yaw axis is positive going into 
the page on the centerline. It is important to note that the PA-1 FTA consists of just the LAS, the CM, and the separation ring. The separation ring is not active and its functionality is not a part of this flight test. The LAS stacked with the CM is known as the Launch Abort Vehicle (LAV). The LAS propulsion system consists of the Abort Motor (AM), the Attitude Control Motor (ACM) and the Jettison Motor (JM). The $\mathrm{AM}$ is a solid rocket motor with four reverse flow nozzles canted outward at $25^{\circ}$ from the motor centerline. The AM generates the thrust necessary to pull the vehicle away from the pad during an abort. The JM is a solid rocket motor having four scarfed nozzles canted at $35^{\circ}$ from the motor centerline. The JM pulls the LAS away from the CM once reorientation is complete. The ACM is a solid rocket motor with eight radially spaced throttleable nozzles placed $45^{\circ}$ symmetrically from the LAS centerline to provide directional control for the LAS. The ACM provides approximately $6500 \mathrm{lbf}$ of commanded omnidirectional thrust for up to 7 s followed by $2500 \mathrm{lbf}$ of commanded omnidirectional thrust from 7 to 27 s. The pad abort flight sequence, which is timer based, is shown in Fig. 2. When a pad abort is initiated, the LAV is pulled away from the pad by the AM, a pitchover sequence is initiated for downrange distance, and the vehicle is stabilized by the ACM. The AM burns out after $5 \mathrm{~s}$ as the ACM continues to stabilize the vehicle. At approximately $11 \mathrm{~s}$ the ACM begins to reorient and restabilize the LAV heat shield forward in preparation for separating the CM from the LAS. When the reorientation maneuvers are complete, the JM fires to separate the CM from the LAS; drogue parachutes are then deployed, followed by the main parachutes.

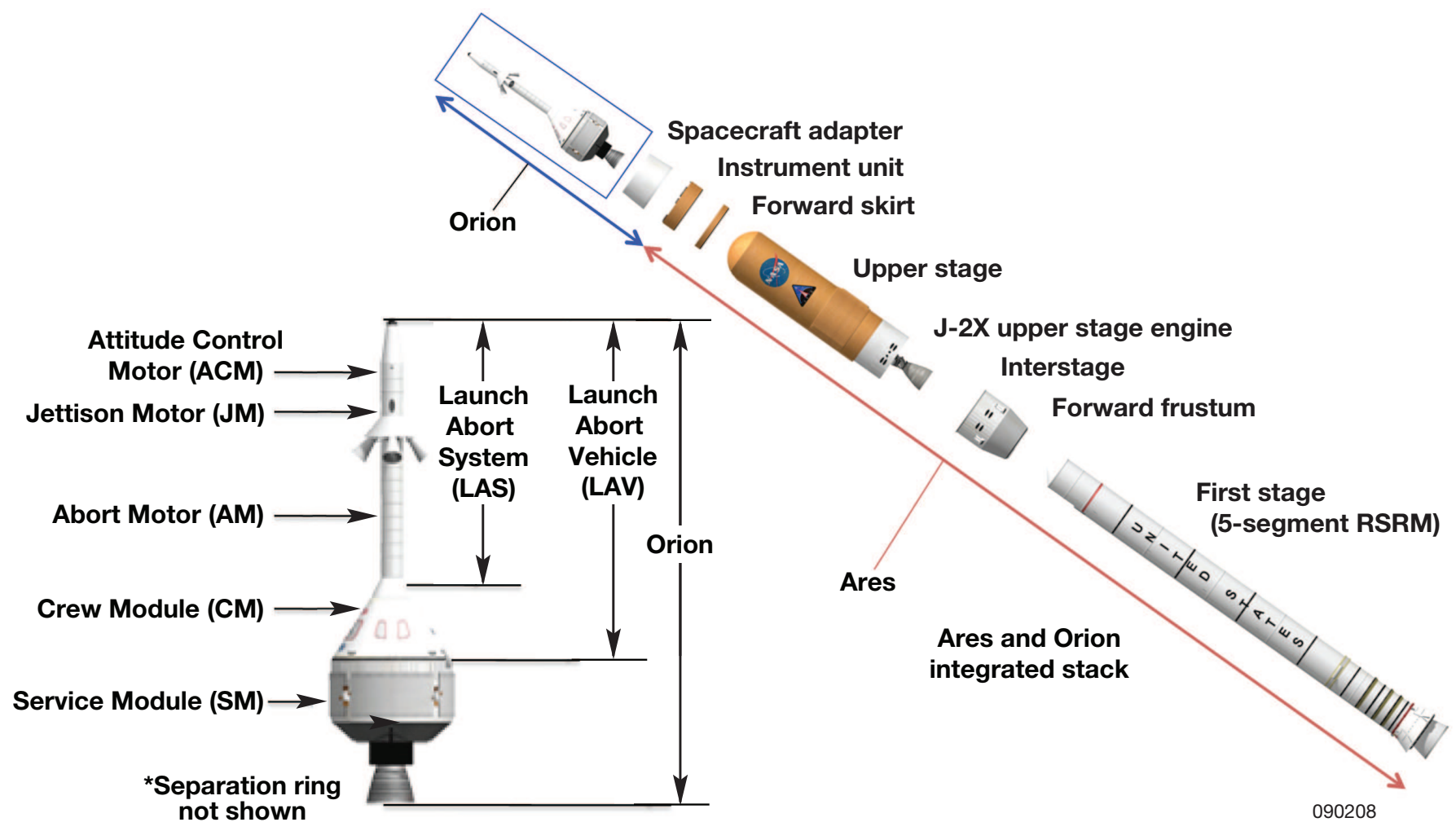

Figure 1. Orion Launch Abort System

\section{B. Guidance, Navigation and Control}

The guidance, navigation and control (GN\&C) systems were designed and tested by Lockheed Martin Space Systems (Denver, Colorado) and Orbital Sciences Corporation (Dulles, Virginia). Due to the proprietary nature of the design, this paper gives an overview of these systems.

Navigation data for the LAS is provided by a Honeywell (Clearwater, Florida) Space Integrated GPS/INS (SIGI) unit mounted in the crew module. It is important to note that the SIGI used in the PA-1 LAS does not have a GPS receiver or a Flush Air Data System (FADS) so all navigation data are purely sensed inertial rates and accelerations. The LAS navigation system uses these rates and accelerations and computes angle of attack, sideslip angle, Mach number, dynamic pressure, downrange distance, body rates and velocities. 


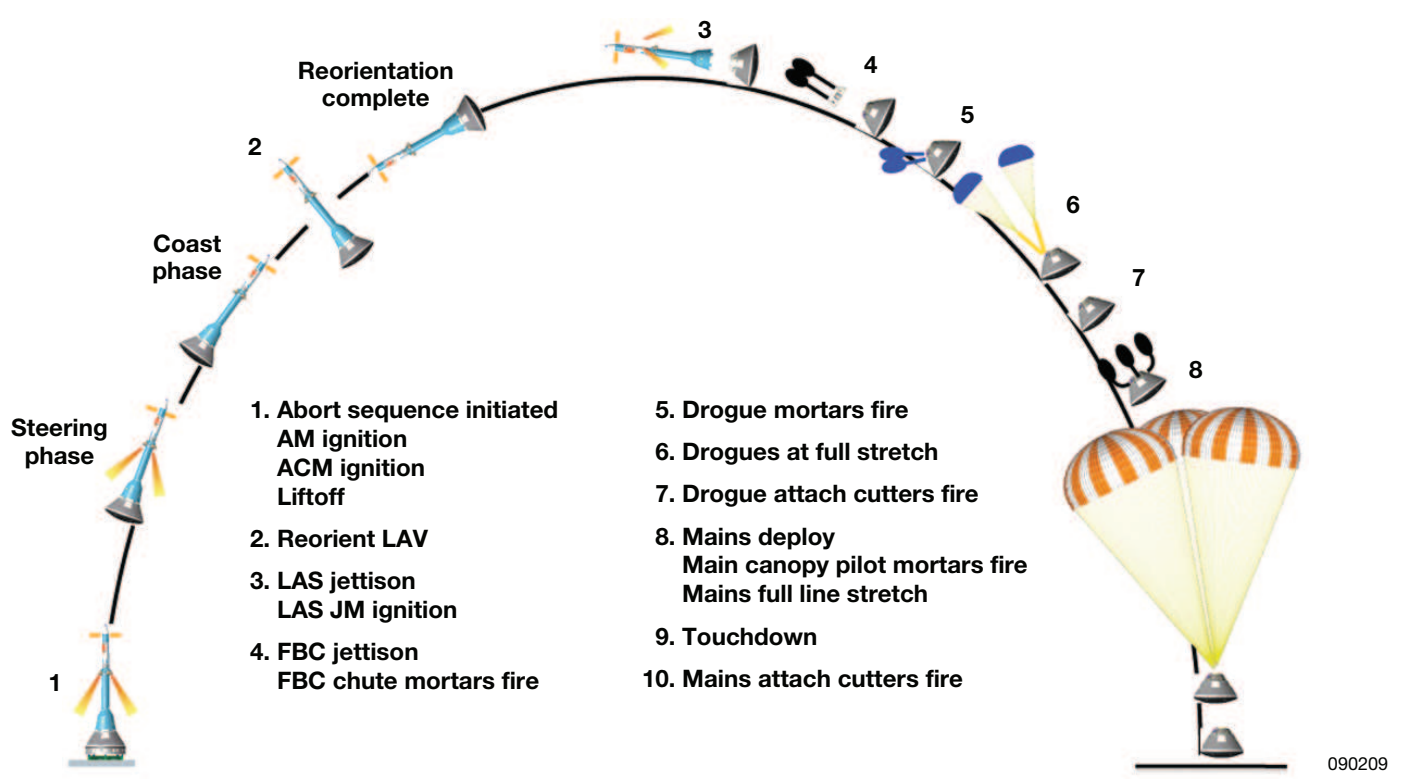

Figure 2. Launch Abort System PA-1 flight profile.

These parameters are then passed on to the control system.

The guidance system is separated into three phases based on time. The steering phase occurs during the first $2 \mathrm{~s}$ of flight. During this phase, steering tables are used to specify a pitch and yaw command for the current time since abort initiation. The next phase, known as the coast phase, occurs from 2 to $10 \mathrm{~s}$ after abort. Angle of attack and sideslip angle commands from predefined tables are compared with the current angle of attack and sideslip angle to generate pitch and yaw commands based on the difference. The reorientation phase takes place from 10 to $21 \mathrm{~s}$ into the flight. The pitch and yaw commands from the coast phase are blended into reorientation commands in terms of angle of attack and sideslip angle. During this phase, angle of attack is commanded based on a table lookup of Mach number and sideslip angle is commanded relative to the commanded angle of attack to reorient the vehicle heat shield forward.

The control system consists of a proportional integral derivative (PID) controller with gains for each axis scheduled by a table lookup of the estimated vehicle mass properties. The difference between the commanded and actual rates are filtered, resulting in pitch and yaw rate commands. These commands are rate limited and then converted to thrust magnitude and direction commands which are sent to the ACM. A Simulink ${ }^{\mathrm{R}}$ AutoCoded software model (The MathWorks, Natick, Massachusetts) of these systems is used in the six-degrees-of-freedom (DOF) simulation known as the Advanced NASA Technology Architecture for Exploration Studies (ANTARES).

\section{Flight-Test Preparation}

\section{A. Flight-Test Operation Overview}

The flight-test team is an integrated team, including members from NASA, Lockheed Martin Corporation, Orbital Sciences Corporation, and WSMR. The team, located inside the Mobile Operations Facility (MOF), leads the countdown to launch. During pre-launch, the health and status of the subsystems is monitored, and a go/no-go recommendation for launch is made based on mission rules and flight placards. Post-launch, the flight-test team monitors the ascent of the FTA for nominal performance.

The MOF is a single-wide trailer structure used as the control room for flight. It houses the subject matter experts from various disciplines, as well as data displays used to support the flight test. The MOF receives the best telemetry data source from both developmental flight instrumentation (DFI) and operational flight instrumentation (OFI) data streams; and displays video from WSMR range sources, tower weather data, and radar data. The MOF has the ability to provide uplink commands to the WSMR UHF system, allowing abort commands to be sent to the vehicle to initiate the pad abort. The range also provides a 924-Mhz 
Doppler radar located near the launch site, which can generate wind profiles every 6 min. The information captured from the Doppler radar will be used in the post-flight reconstruction of the trajectory along with radar tracks of the LAS and CM, the OFI data recorded from the SIGI during flight, and the DFI data recorded from the flush air data system located in the nose cone of the LAS and the heat shield on the bottom of the CM.

\section{B. Hardware Testing}

A three-tiered approach will be taken in testing the LAS hardware. With each increasing tier, the fidelity of the tests increases due to the increasing amount of flight hardware used in the testing. The first tier of hardware testing consists of using a CM-like structure in which vehicle avionics such as the Vehicle Management Computer (VMC) and the SIGI will be mounted as in the PA-1 CM. Testing is then performed on flight software to determine if there are any issues such as dropped frames. A PA-1 trajectory will then be run using a mix of real hardware and software emulation. The data from this test will then be analyzed by the flight dynamics team to ensure that the trajectory and sequencing were executed properly. After the first tier of testing, procedures will be refined for the second and third tiers of testing. The second tier of testing will be performed at NASA Dryden using the PA-1 CM. The same tests from the first tier will be run during the second tier on the PA-1 CM. If no anomalies are found, the PA-1 CM will be shipped to WSMR and integrated with the LAS hardware. The third tier of tests will be run again on the integrated hardware and checked out for flight.

\section{Real-Time Data Analysis}

Each discipline engineer in the MOF will monitor the telemetry from real-time displays using the Symvionics (Arcadia, California) Interactive Analysis and Display Software (IADS ${ }^{\mathrm{R}}$ ). The IADS displays are custombuilt for each discipline's needs to include warning and alarm indicators for mission-critical and safety-of-flight parameters. Fig. 3 below shows a screen shot of one of the GN\&C screens currently under development. It includes digital strip charts, crossplots and ActiveX ${ }^{\mathrm{R}}$ (Microsoft Corporation, Redmond, Washington) controls that indicate the health and performance of the LAS during the flight.

An interface routine has also been developed to run simulation data through the IADS. This feature is invaluable for pre-flight and post-flight data analysis to compare nominal or dispersed predictions with actual flight-test data. Additionally, on the day of launch, the predicted trajectories for the LAV, LAS and $\mathrm{CM}$ will be visible on a crossplot with the actual trajectories to monitor deviations.

\section{Simulation and Analysis}

\section{A. The Advanced NASA Technology Architecture for Exploration Studies}

\section{Overview of ANTARES}

The Advanced NASA Technology Architecture for Exploration Studies (ANTARES) simulation is an Orionspecific simulation built using the Trick ${ }^{\mathrm{R}}$ (L-3 Communications Holdings, Inc., New York, New York) simulation toolkit and is written in the $\mathrm{C}++$ computer language for Linux platforms. The collection of common subsystem models for compilation of the Orion mission-specific simulation is handled by Trick ${ }^{\mathrm{R}}$ for ANTARES. The ANTARES simulation can modify parameters after the compilation of the simulation through the use of input files. These input files can be used to modify mass properties, parachute timing, etc. for an individual run or in a set of trajectories such as a dispersed Monte Carlo set. ${ }^{1}$

The ANTARES simulation provides simulated objects for the CM, LAS, and SM. The main body object, from the full stack to only the CM, is tracked in 6 DOF and all objects jettisoned from the main body are tracked in 3 DOF. The ANTARES simulation includes a variety of subsystem models that can be exchanged using the Trick ${ }^{\mathrm{R}}$ compilation routine. The subsystem models include atmospheric models such as the Global Reference Atmospheric Model (GRAM), aerodynamic models, and a Simulink ${ }^{\mathrm{R}}$ AutoCoded version of the GN\&C model. The ANTARES simulation also provides a set of plotting tools in addition to the simulation modeling environment, which allows for quick analysis of the data after the simulation run.

The variety of subsystem models, parameter modification, Monte Carlo analysis capabilities, and data analysis tools give ANTARES the versatility to create specific simulations for flight test. The ANTARES simulation was extensively used in the analysis described in this paper. ${ }^{2}$ 


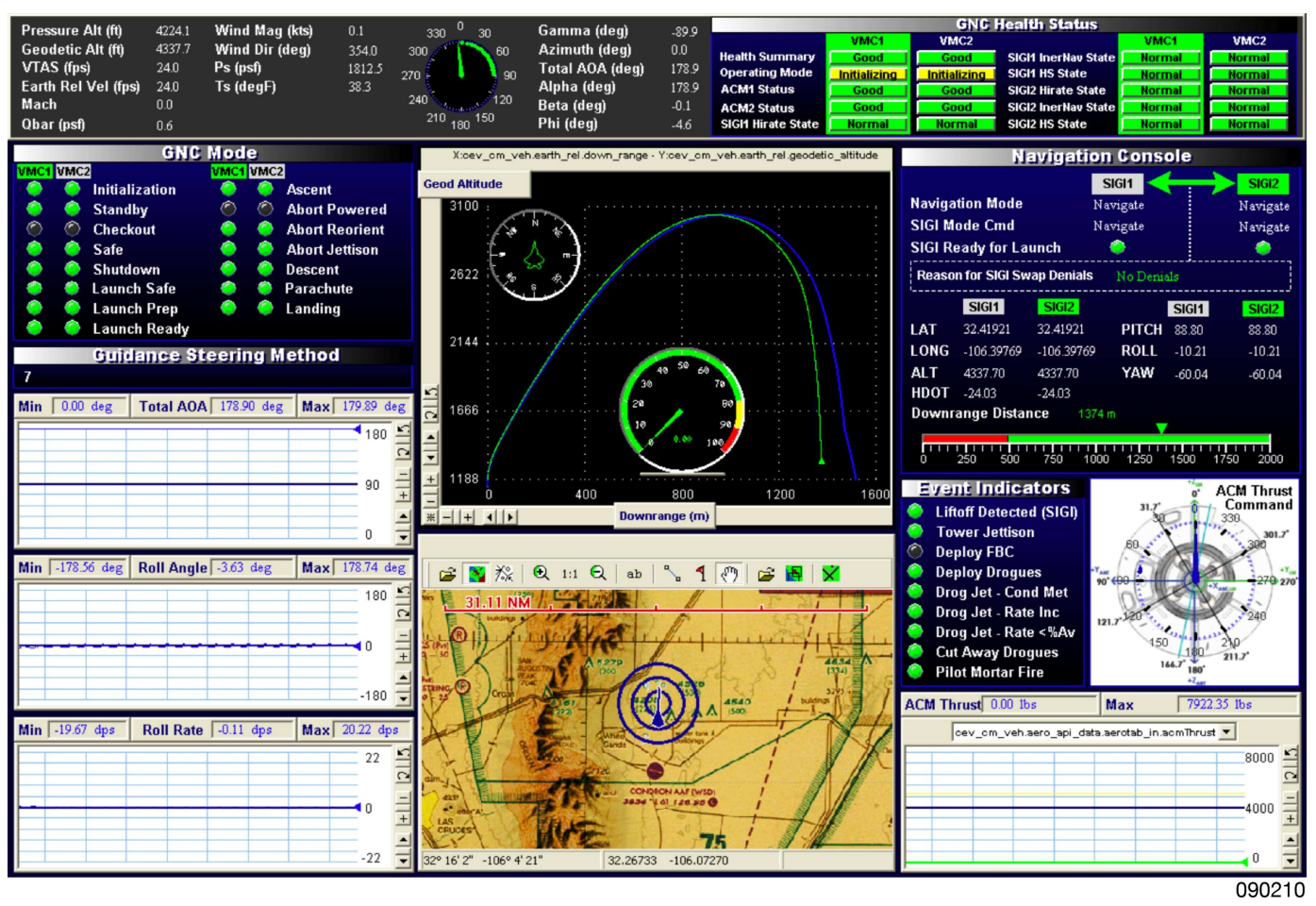

Figure 3. Screen shot of real-time display.

\section{The ANTARES Atmospheric Model}

The NASA Dryden Flight Research Center has performed a significant amount of work to determine the most accurate atmospheric models for use with PA-1. Currently, winds and other atmospheric conditions along the launch trajectory are modeled in ANTARES with GRAM-99 Version 3. The GRAM includes several empirical models, however, for the PA-1 ANTARES simulation, the 2006 WSMR Range Reference Atmosphere (RRA) is used. The RRA provides a much more accurate representation of the launch sites monthly climatological conditions, whereas the GRAM models are more generalized. The RRA provides tabulations for monthly and annual means, standard deviations, skewness, and observation counts for pressure, temperature, density, water vapor pressure, virtual temperature, dewpoint temperature, and winds (zonal and meridional wind components and the linear correlation coefficient between the wind components). These statistical parameters are tabulated at the station elevation, at 1-km intervals from sea level to $30 \mathrm{~km}$, and at 2-km intervals from 30 to $70 \mathrm{~km}$, which is well beyond the altitude range for PA-1.

Mean climatological values eliminate natural peaks and troughs caused by daily diurnal effects or atmospheric wave patterns, such as turbulence, thus, ANTARES requires a perturbation model to restore these dispersions; this is accomplished with GRAM-99. While the diurnal effects are not accounted for in GRAM-99, the effects of small-scale (turbulence, mesoscale storms, gravity waves, etc.) and large-scale (baroclinic/Rossby waves, cyclones and anticyclones, tides, etc.) wave patterns are statistically modeled. ${ }^{3}$

The amalgamation of GRAM-99 Version 3 and the 2006 WSMR RRA were verified and validated in ANTARES at NASA Dryden. Climatological data in ANTARES were verified as having been processed correctly by the GRAM-99 subroutine, and the RRA data were validated to sufficiently model measured atmospheric parameters from actual range data. The validation was performed by comparing atmospheric parameters from fully-dispersed ANTARES Monte Carlo simulations with Vaisala rawinsonde balloon profiles launched from locations around the WSMR range from years 1992 to 2006 (54 locations and 8,847 total launches). The density, pressure, temperature, and meridional wind all show relatively good correlation. Figure 4 shows a plot of the WSMR ballon data and the dispersed winds from the GRAM-99 and 2006 WSMR RRA models for the east wind. The balloon data exhibits stronger winds blowing east up to 1,300 $\mathrm{ft}$ AGL. The means from the RRA and the balloon data are very similar, but the difference is driven by 3-sigma winds that vary by nearly $20 \mathrm{ft} / \mathrm{s}$ at lower altitudes. The 1983 and $2006 \mathrm{WSMR}$ RRAs were used 
in ANTARES for separate validation studies. Both showed similar correlation to the balloon data, although the 2006 WSMR RRA was found to be slightly more accurate. Dryden has requested an updated RRA from the Air Force Combat Climatology Center for future simulations because the 1983 and 2006 RRAs appear to be under-predicting winds.

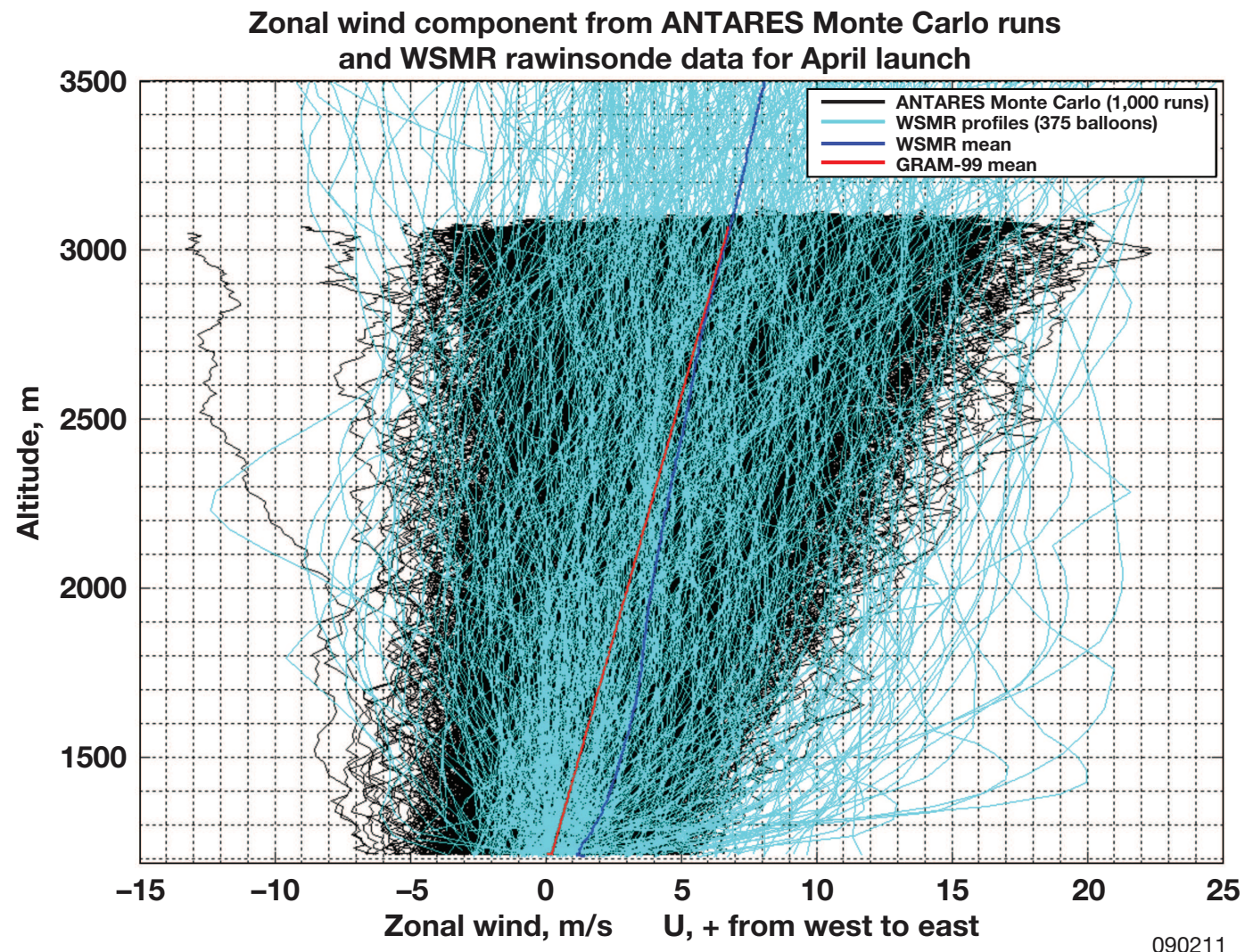

Figure 4. East wind comparison between WSMR ballon data and dispersed GRAM-99 and 2006 WSMR RRA data.

The importance of a realistic atmosphere model for launch abort simulations cannot be overstated. Not only does the atmospheric model affect the trajectory and landing footprint, it also affects the highly sensitive LAS jettison phase of flight. To meet the LAS jettison conditions, wind placards will be determined from Monte Carlo dispersions of the atmospheric model to ensure specification requirements are met with a $99.73 \%$ probability of success on the day of flight.

\section{The ANTARES Aerodynamic Model}

The aerodynamic model implemented in ANTARES was commissioned by the CEV Aerosciences Project (CAP) to leverage a confluence of aerodynamic expertise in analytical and experimental techniques at the NASA Johnson Space Center (Houston, Texas), the NASA Ames Research Center (Moffett Field, California), and the NASA Langley Research Center (Hampton, Virginia). The model consists of two basic components: an aerodynamic database and a C-coded Application Programming Interface (API) to access the database and render coefficients. ${ }^{4}$ The NASA Dryden contribution to this area involved V\&V of the CAP API in ANTARES. For PA-1 flight testing, a database specific to the PA-1 flight test vehicle was used in ANTARES; future abort flight tests and Orion missions will use a different aerodynamic database.

Many of the complex configuration and flowfield aerodynamic phenomena inherent to the LAV, LAS and $\mathrm{CM}$ are modeled in the PA-1 aerodynamic database, including basic aerodynamic coefficients for all configurations, dynamic damping terms for both the LAV and CM configurations, plume effects from the $\mathrm{AM}$ and ACM, and uncertainty levels with uniform or Gaussian distribution. Currently, plume effects for the JM are not included, nor are close-proximity effects as the LAS is jettisoned from the CM. 
The CAP API was implemented within the ANTARES simulation environment using an aerodynamic executive for interface routines to include coordinate system transformations, unit conversions, and other computations of input variables required by the API. From these inputs, the API calculates coefficients, forces, and moments that are returned to ANTARES to compute the equations of motion and equations for any frame transformations. ${ }^{5}$

\section{B. Monte Carlo Analysis}

Monte Carlo analysis methods are a class of statistical simulation methods that use random samples of inputs to explore the behavior of complex systems. The NASA Dryden Flight Research Center has a long history of using Monte Carlo analysis in flight test programs for aircraft such as the X-33 ${ }^{6}$ and the X-43. ${ }^{7}$ Monte Carlo analysis is used extensively in the PA-1 flight test program, from the design of the LAS PA-1 trajectory to testing the robustness of the control system. For example, Figure 5 shows data from a 5000-run Monte Carlo simulation of angle of attack $\alpha$ vs. time during a PA-1 trajectory simulation in which certain models such as aerodynamics, mass properties, and controller were dispersed. Since there is plenty of control margin and the ACM is not saturated during the flight the controller performance is very good. Additionally, there are no uncommanded vehicle flip-overs after abort initiation, and reorientation was successfully achieved in all 5000 runs.

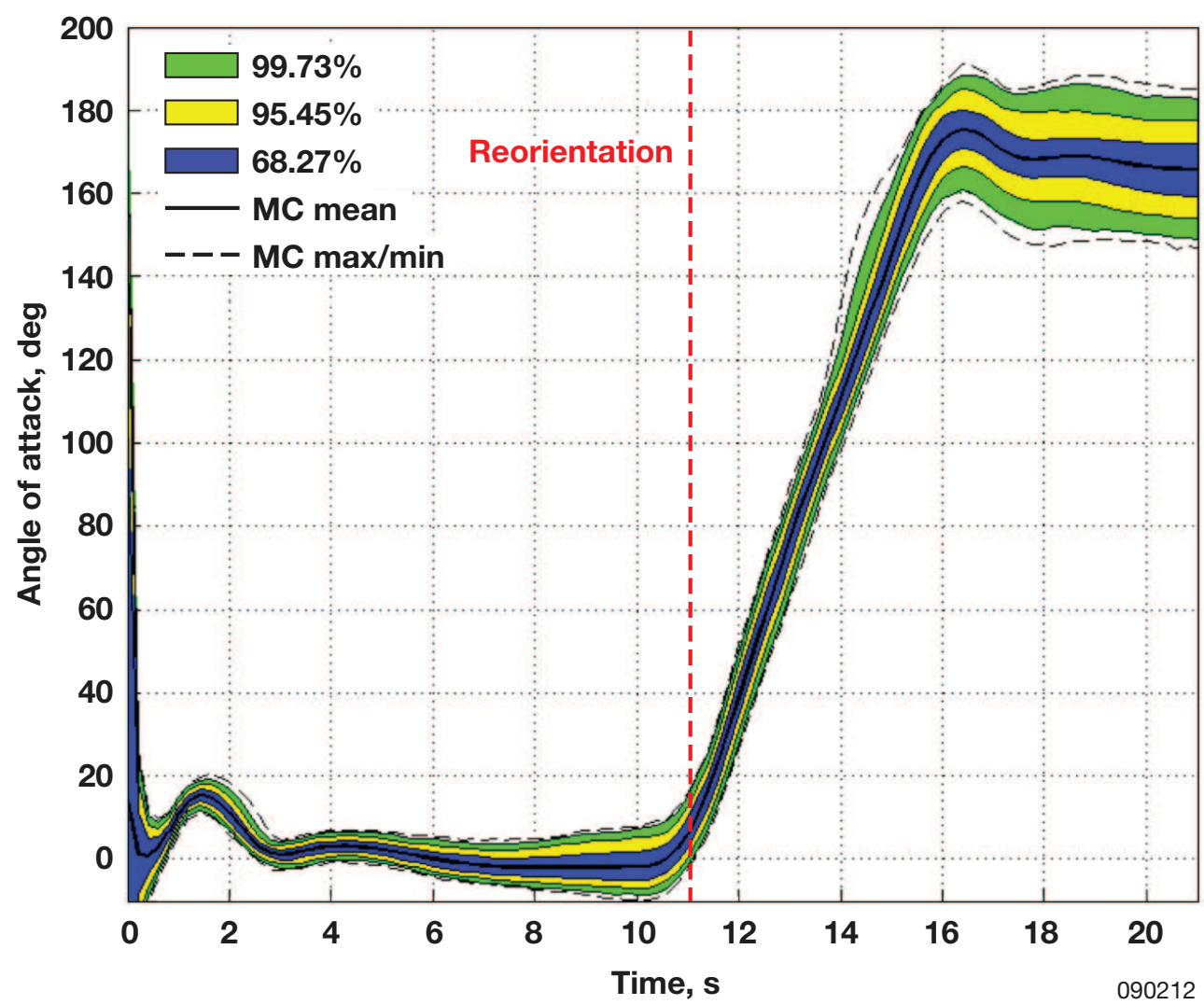

Figure 5. Controller performance analysis.

Another use of Monte Carlo analysis was examining the sensitivity of the LAV controller to larger bounds of the mass properties dispersions. The LAS jettison constraints were derived based on the dynamic requirements of the parachute design team. Equations 1 and 2 shows the LAS jettison ellipsoid and roll rate constraints. Satisfying these constraints improves the likelihood of successful reorientation and deployment of the drogue and main parachutes. In these equations, $\alpha_{t j e t t}, \beta_{t j e t t}, p_{t j e t t}, q_{t j e t t}$ and $r_{t j e t t}$ are the angle of attack, sideslip angle, roll rate, pitch rate, and yaw rate at the time of jettison. This study is essentially examining how the system behaves with increased uncertainty in the mass properties and the resulting effect on the trajectory. Figure 6 shows a plot of each of the two constraints after the mass properties dispersions 
were expanded to increase the bounds. There were 0 violations of the jettison ellipsoid constraint. Increasing the bounds on the dispersions did not increase the number of violations, as is shown in Table 1. Therefore, mass properties do not have a significant effect on the LAS jettison ellipsoid. Despite this conclusion, extensive mass properties testing will be performed to provide engineers with weight, center of gravity (c.g.), and uncertainties of the LAS mass properties model. Although mass properties do not affect the LAS jettison ellipsoid, these data provide critical input to other subsystems groups, such as structures and aerodynamics.

$$
\begin{gathered}
\left(\frac{\alpha_{t j e t t}}{34^{\circ}}\right)^{2}+\left(\frac{\beta_{t j e t t}}{34^{\circ}}\right)^{2}+\left(\frac{\sqrt{q_{t j e t t}^{2}+r_{\text {tjett }}^{2}}}{21 \frac{\circ}{s}}\right)^{2} \leq 1 \\
p_{\text {tjett }} \leq 45 \frac{\circ}{s}
\end{gathered}
$$

Table 1. Sensitivity of LAS jettison constraints to mass properties.

\begin{tabular}{lccc} 
Test case number & 3-sigma dispersions & Number of failures & Dispersed parameters \\
\hline 1 & $50 \%$ increase from nominal & 0 & weight, c.g.'s, and inertias \\
2 & $200 \%$ increase from nominal & 0 & c.g.'s and inertias \\
\hline
\end{tabular}
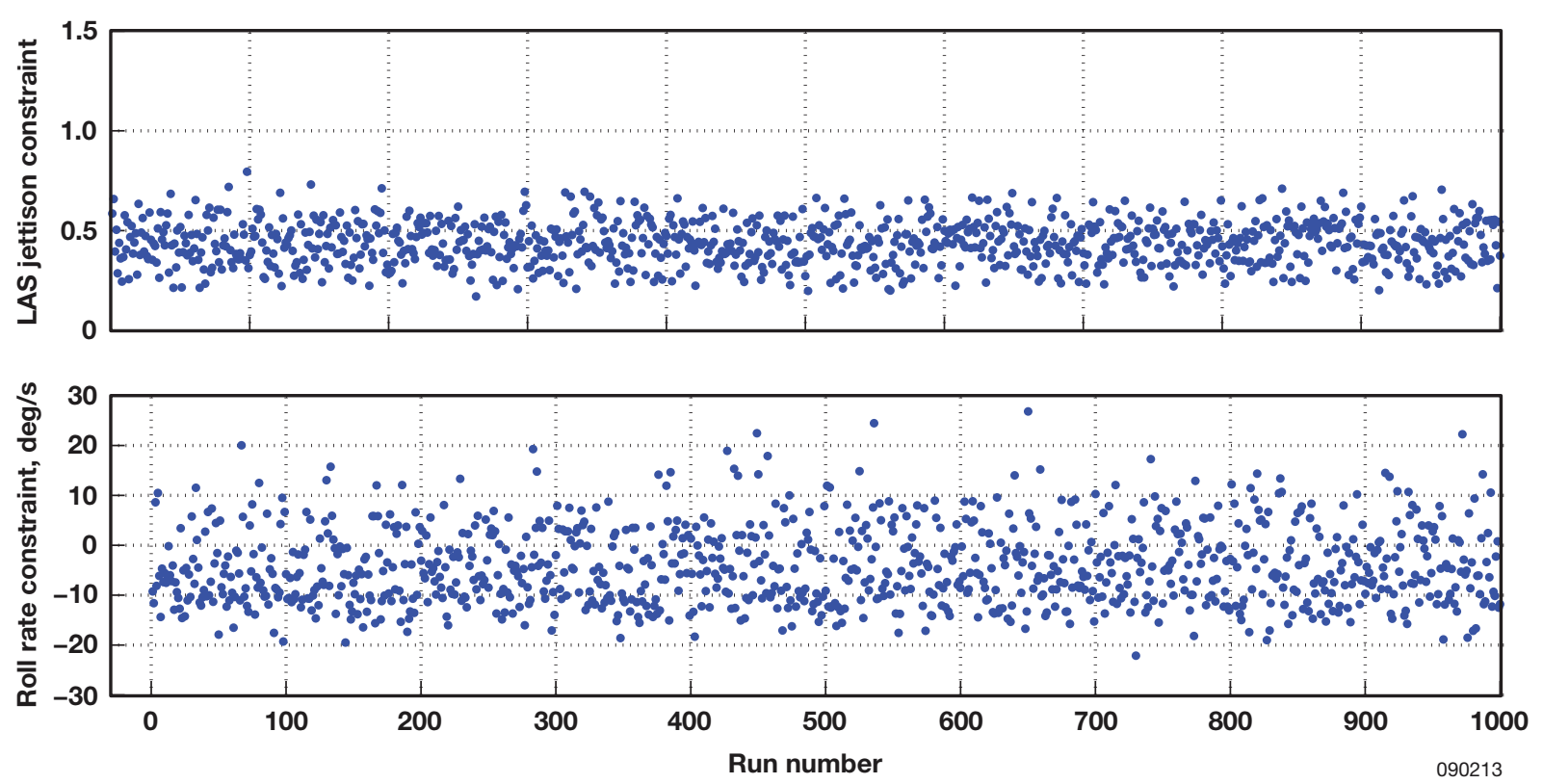

Figure 6. Launch Abort System jettison Monte Carlo analysis results with increased uncertainty in mass properties dispersions.

A parametric Monte Carlo analysis was conducted on the aerodynamic database to observe the effects of off-nominal uncertainties. In these simulations, aerodynamic coefficient uncertainty factors were dispersed to explore the impact on specification requirements and control margins. Not only did this help quantify risk by projecting likely failure scenarios, it also allowed engineers to visualize the influence of the aerodatabase coefficients and derivatives on the trajectory and the control system. As an example, Figure 7 shows the effect on the ACM from dispersing the yawing moment coefficient. This plot indicates that there is region (blue to yellow) where the yawing moment uncertainty factor scales linearly to the required thrust from the ACM. There is a point shown in red, however, where the uncertainty is too high and the ACM saturation limits are reached. Using Monte Carlo analysis in this way enables the test team to explore off-nominal behavior and take steps to eliminate or mitigate these problems. 


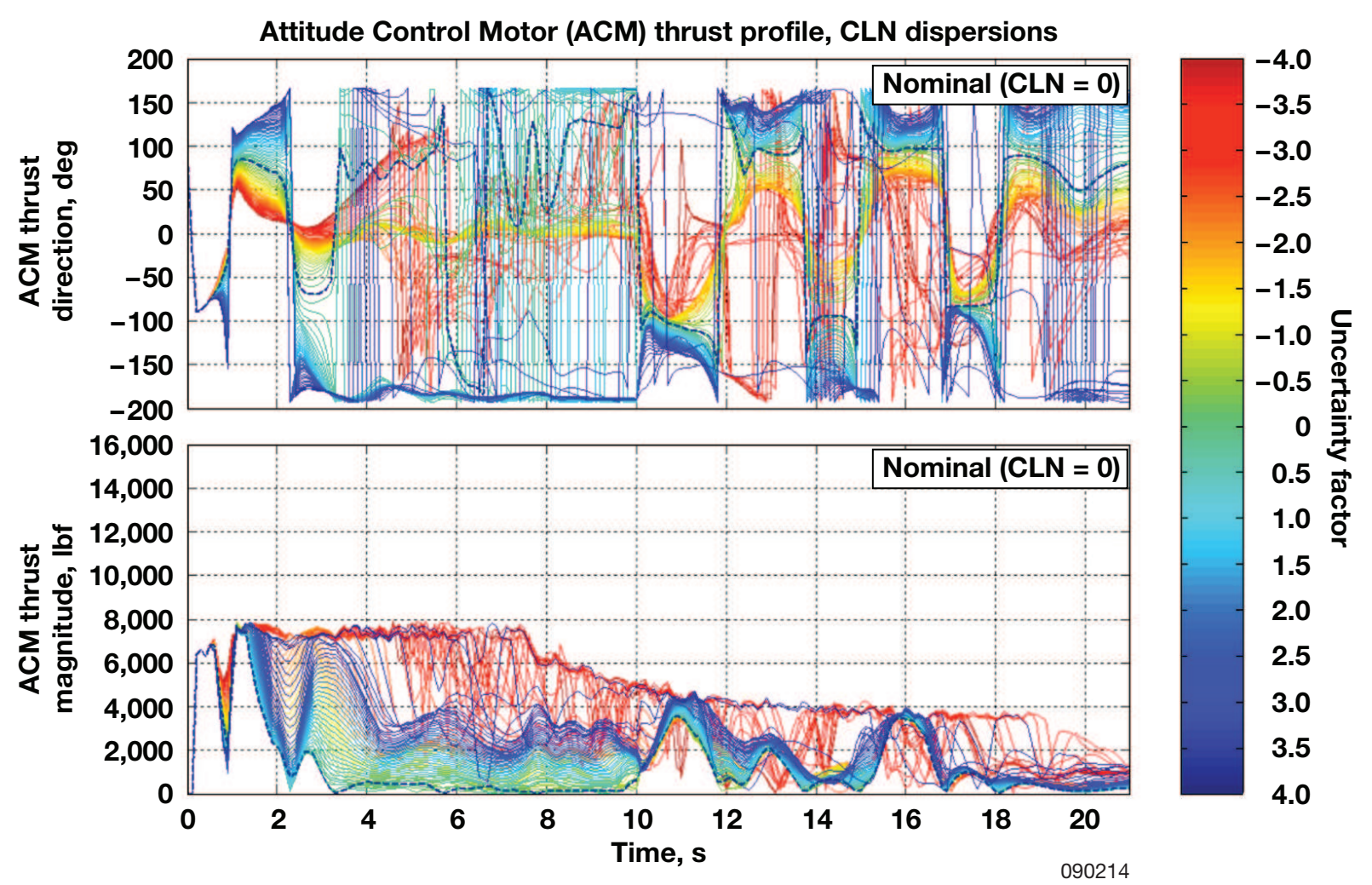

Figure 7. Attitude Control Motor commands resulting from dispersed yawing moment coefficient.

Additionally, Monte Carlo analysis was used to generate data sets for a data mining technique (AutoBayes and TAR3) to provide insight into characteristics driving LAS jettison conditions. Given a set of data, AutoBayes characterizes its structure to identify nominal and off-nominal behavior. This is then passed on to the TAR3 (Treatment Learner) algorithm which, when given a data set, tries to find input parameter ranges that produce better results. Essentially this technique provides optimal ranges and margins for inputs as well as identifying sensitivities between inputs and outputs. ${ }^{8}$ Figure 8 shows a plot of altitude vs. longitude and latitude of the LAV trajectory. The key drivers for a successful trajectory are meeting the touchdown velocity and downrange distance criteria. The trajectories that met all the criteria are shown in blue while the trajectories that failed all the criteria are shown in red. This analysis identified atmospheric winds to be key drivers in the success of LAS jettison and reorientation. These results were used to drive a new trajectory design that mitigates the sensitivity of the vehicle to winds, and to create mission rules that will ensure the highest probability of success.

\section{Conclusion}

The Pad Abort 1 flight test of the Launch Abort System (LAS) is a major milestone in the Constellation program. This paper presents a brief overview of the LAS pad abort configuration and the NASA Dryden Flight Research Center (Edwards, California) guidance, navigation, and control efforts currently underway in preparation for the flight test. The methods used in analyzing the trajectory along with the flight test operations were examined. From these efforts the following conclusions can be made: the controller performance is very robust, however, winds are a key driver in a successful LAS jettison and reorientation. As such, wind limits will be used on launch day to mitigate this risk and to ensure highest probability of success. The LAS performance during jettison is not sensitive to mass properties but testing will nevertheless be performed to provide the highest fidelity possible to the simulation and modeling of this critical maneuver. Lessons learned from the pad abort test will be propagated through the Constellation Program and will help avoid costly mistakes and delays. These steps, as well as recent program successes toward the first pad abort test, show great promise for the future of Orion and American space exploration. That said, the true 


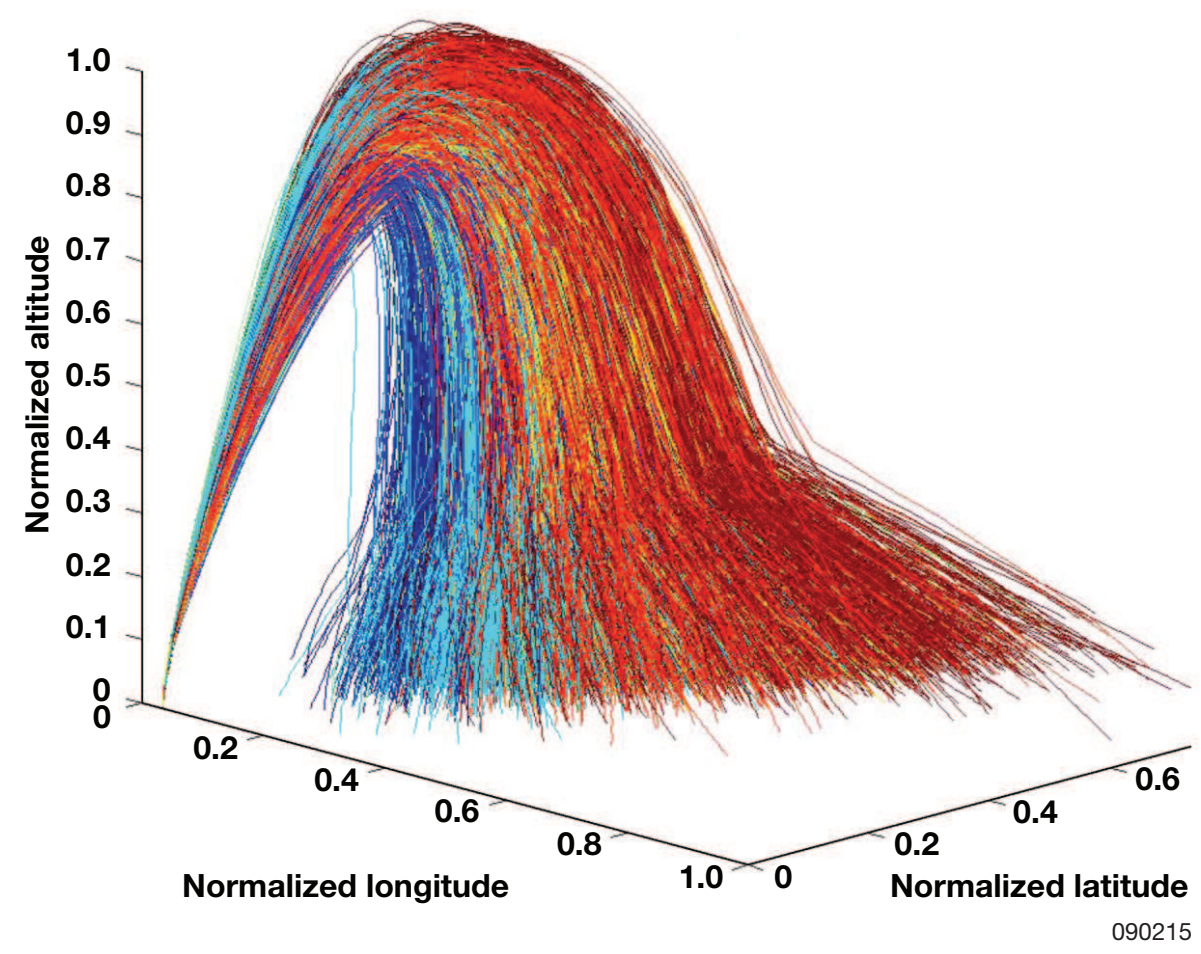

Figure 8. Monte Carlo analysis results of LAS trajectory.

indicator will be the launch itself.

\section{Acknowledgements}

The authors acknowledge the following people for their contributions to this paper: Karen Gundy-Burlet of the NASA Ames Research Center (Moffett Field, California) for her assistance with data mining techniques and sensitivity analysis; Roger Beck of AMA, Inc. (Hampton, Virginia) for his assistance with controller performance analysis; and Joe Gamble of Lockheed Martin Corporation (Bethesda, Maryland) for his work on and explanation of the LAS jettison ellipsoid design.

\section{References}

\footnotetext{
${ }^{1}$ Hoelscher, B., "Orion Entry, Descent, and Landing Simulation," AIAA, 2007.

${ }^{2}$ Acevedo, A., Arnold, A., Othon, W., and Berndt, J., "ANTARES: Spacecraft Simulation for Multiple User Communities and Facilities," AIAA, 2007.

${ }^{3}$ Justus, C. G. and Johnson, D. L., "The NASA/MSFC Global Reference Atmospheric Model 1999 Version (GRAM-99)," Tech. Rep. NASA TM-1999-209630, National Aeronautics and Space Administration, 1999.

${ }^{4}$ Greathouse, J., Kirk, B., Lillard, R., Truong, T., Robinson, P., and Cerimele, C., "Crew Exploration Vehicle (CEV) Crew Module shape selection analysis and CEV Aeroscience Project Overview," AIAA, 2007.

${ }^{5}$ Robinson, P. E. and Thompson, J. A., "Formulation of the Orion Aerodynamic Database, Revision 0.51.2," Tech. Rep. NASA EG-CEV-06-37, National Aeronautics and Space Administration, 2008.

${ }^{6}$ Williams, P., "A Monte Carlo Dispersion Analysis of the X-33 Simulation Software," AIAA, 2001.

${ }^{7}$ Bahm, C., Baumann, E., Martin, J., Bose, D., Beck, R., and Strovers, B., "The X-43A Hyper-X Mach 7 Flight 2 Guidance, Navigation and Control Overview and Flight Test Results," AIAA, 2005.

${ }^{8}$ International Symposium on Artificial Intelligence, Parametric Analysis of Antares Re-Entry Guidance Algorithms using Advanced Test Generation and Data Analysis, 2008.
} 